\title{
Dörfliche Infozentren in Südindien:der Mensch vor Werkzeug
}

Michel Egger

\section{(2) OpenEdition Journals}

Electronic version

URL: http://journals.openedition.org/sjep/532

DOI: $10.4000 /$ sjep.532

ISSN: 1663-9677

\section{Publisher}

Institut de hautes études internationales et du développement

\section{Printed version}

Date of publication: 1 novembre 2003

Number of pages: $85-87$

ISSN: $1660-5926$

Electronic reference

Michel Egger, « Dörfliche Infozentren in Südindien:der Mensch vor Werkzeug », Schweizerisches Jahrbuch für Entwicklungspolitik [Online], 22-2 | 2003, Online erschienen am: 10 Juni 2010, abgerufen am 08 September 2020. URL : http://journals.openedition.org/sjep/532 ; DOI : https://doi.org/ 10.4000/sjep.532 


\title{
Dörfliche Infozentren in Südindien: der Mensch vor Werkzeug
}

\author{
Michel Egger*
}

V

illianur. Eine grosse Stadt $13 \mathrm{~km}$ westlich von Pondicherry (Tamil Nadu), Südindien. Hier befindet sich der $H u b$, das Herz des 1998 von der Stiftung Swaminathan $^{1}$ gegründeten Netzwerkes ländlicher Infozentren. Zehn Dörfer im Umkreis von $25 \mathrm{~km}$ sind heute durch ein Doppelsystem - mit und ohne Kabel - für die Übertragung von digitalen Daten und Voice Mails verbunden. Verschiedene technologische Mittel werden - der jeweiligen Situation entsprechend angepasst eingesetzt: PC, Telefon, VHFDuplex-Sprechfunk, Webcams. Auch der Strom kommt aus zwei Quellen: Solarstrom (60\%) und konventionelle elektrische Energie (40\%). Dies ist eine Notwendigkeit, denn Strompannen sind häufig und nicht alle Dörfer verfügen über Strom.

\section{Entscheidende Informationen}

Heute ist der Hub ,voll im Schwung“. Jeden letzten Samstag des Monats findet ein Treffen der Freiwilligen statt, welche die Infozentren betreiben. Rund dreissig junge Leute - gleich viele Frauen wie
Männer - versammeln sich auf der Dachterrasse. Sie berichten über Aktivitäten, Probleme und Projekte. Ein Bericht folgt dem anderen, unterbrochen von Fragen und Applaus.

Die Erzählungen illustrieren schön, wie die Zentren den Alltag der Menschen verändern. Diese finden hier entscheidende Informationen. Da hat ein Fischer eine Stelle als Feuerwehrmann gefunden. Dort wurde eine Möglichkeit entdeckt, einen Parasiten auszurotten, der die Zuckerrohrernte bedrohte. Die Tagelöhner in der Landwirtschaft erfahren die Mindestlöhne und können weniger ausgenutzt werden. Die Produzenten halten sich auf dem Laufenden über die Marktpreise und sind nicht mehr von den Zwischenhändlern abhängig. Und dank der Wettervorhersagen hat sich die Sicherheit der Fischer auf dem Meer erhöht. Die Infozentren haben zahlreiche Hilfsprogramme der Regierung aufgeschaltet und machen die verschlungenen Wege der öffentlichen Verwaltung transparenter. Damit sind sie auch zu Katalysatoren für kollektive Aktivitäten geworden: Selbsthilfegruppen für Frauen, Pro-

* Journalist und Koordinator für Entwicklungspolitik bei der Arbeitsgemeinschaft der Hilfswerke Swissaid/Fastenopfer/Brot für alle/Helvetas/Caritas/HEKS, Schweiz.

1990 in Chennai vom „Vater der grünen Revolution“ Indiens gegründet, dessen Namen sie übernommen hat. Die M. S. Swaminathan Research Foundation ist eine Pionierinstitution der wissenschaftlichen Forschung und der angewandten Technologie für nachhaltige Entwicklung. <www.mssrf.org>. 
jekte für Kleinstkredite und gemeinschaftliches Sparen, Gründung von Kleinstunternehmen (Champignonzucht, biologische Schädlingsbekämpfung, Reinigungsprodukte usw.).

\section{Die Schlüssel des Erfolgs}

Das Versuchsprojekt erhielt mehrere Auszeichnungen und wird von der Schweizer Direktion für Entwicklung und Zusammenarbeit (DEZA) über miteinander verbundene Netzwerke (Global Knowledge Partnership und OneWorld) indirekt unterstützt. Es gilt heute als Modell nicht nur in Indien. Welches sind die Schlüssel seines Erfolgs? Die verfügbaren Evaluationsstudien ${ }^{2}$, die Diskussionen mit den Verantwortlichen in Chennai und die Freiwilligen im Feld weisen auf vier Elemente hin:

1) Mensch vor Technik. Bereits 1992 widmete die Stiftung Swaminathan ihr Jahresseminar - mit Fachleuten und Abgeordneten aus den Dörfern den Informations- und Kommunikationstechnologien (IKT). Es hatte den herausfordernden Titel ,Reaching the Unreached“. „Wenn man will, dass die IKT zu echten Instrumenten der nachhaltigen Entwicklung werden", erklärt S. Senthilkuraman, der Direktor des Informatikzentrums der Stiftung in Chennai, „muss man sich nicht zuerst auf das Werkzeug, sondern vielmehr auf den Menschen konzentrieren. Man darf nicht eine Technologie anwenden, einfach weil es sie gibt, sondern, weil sie wirkliche Vorteile bringt. Sie ist also nicht Zweck, sondern ein Mittel, das an Kontext, Bedürfnisse und Fähigkeiten der lokalen Gemeinschaften angepasst werden muss - und nicht umgekehrt." Deshalb führte die Stiftung vor der Einführung des Programms 1997 in der Region mehrere partizipative Studien durch, um den Alphabetisierungsgrad (42\%), den Zustand der Telekominfrastrukturen, die Kommunikationsgewohnheiten, die Art der nützlichen Informationen usw. festzustellen.

2) Partizipatives Vorgehen. „Die Beziehung zwischen der Stiftung und der lokalen Gemeinschaft ist nicht eine ,Geber-Empfänger-Beziehung“, sondern eine Partnerschaft im Aufbau“, hält S. Senthilkumaran fest. „Wir haben nur dort ein Projekt gestartet, wo die Dorfbewohnerinnen und -bewohner dies wünschten und sich daran beteiligen. Wir wollen, dass sie sich wirklich als Eigentümer dieser Zentren fühlen." Das Prinzip dieser Zusammenarbeit ist einfach: Die Stiftung liefert das Material, die technische Betreuung, die Ausbildung der Leitenden. Die Gemeinschaften ihrerseits müssen ein passendes öffentliches Lokal zur Verfügung stellen, die Strom- und Telefonkosten übernehmen und die freiwilligen Leiterinnen und Leiter bestimmen.

3) Weiterbildung und Empowerment,
insbesondere der Frauen. „Beim ganzheitlichen Vorgehen der Stiftung stehen die Armen, die Natur und die Frauen im Zentrum“, erläutert K.G. Rajamohan, der für den $H u b$ von Villianur verantwortlich ist. „Die Erfahrung zeigt: Wenn die Frauen von einer Technologie profitieren, profitiert die ganze Familie. Bei den Männern ist das nicht unbedingt so." Heute fangen die Freiwilligen selber an, auszubilden: Drei Infozentren 
beteiligen sich nun am Pilotprojekt des Erziehungsdepartements von Pondicherry, das die Einführung der Informatik in den Landschulen zum Ziel hat.

4) Betonung der lokalen Inhalte. „Das Projekt hat nur dann einen Sinn und eine Chance, wenn es Informationen liefert, welche den Leuten helfen, ihre Situation zu verbessern. Sonst verlieren diese schnell das Interesse", erklärt Rajamohan. Es gibt zwei Arten von Informationen: Aktualität und Hintergrundwissen. Bei ersterer geht es namentlich um die Wettervorhersagen, die Marktpreise, die Stellenangebote, den öffentlichen Verkehr, die medizinischen Dienste, die Kleininserate usw. Das Hintergrundwissen betrifft die Hilfsprogramme der Regierung, die gelben Seiten, die Gesundheit der Frauen, die landwirtschaftlichen Praktiken usw. Die Informationen werden von Freiwilligen aus den Dörfern und den Mitarbeitenden des Hubs zusammengetragen. Die Quellen reichen von Websites (wie jene der amerikanischen Marine für die Höhe der Wellen im Golf von Bengalen) über die lokale Presse bis zu jungen Reportern, welche die Märkte „abklappern“. Vor allem wird den Informationen ein „Mehrwert“ beigefügt, bevor sie aufgeschaltet werden: „Es geht nicht nur darum, die Infor- mationen zu sammeln und zu verbreiten. Sie müssen auch verarbeitet, angepasst und in das lokale Wissen integriert werden“, präzisiert Rajamohan. An einigen Orten, so im Fischerdorf Veerampattinam, gibt man die wichtigen Informationen und die Wettervorhersagen über Lautsprecher bekannt.

\section{Finanzielle Unterstützung nötig}

Zwei grosse Herausforderungen bleiben. Als Erstes müssen die Vorurteile beseitigt werden. „Alle können zu uns kommen, ohne Unterschied, und die Infozentren werden immer bekannter und sind immer besser besucht. Aber noch glauben viele, dass sie nur für die Gebildeten oder gewisse Kasten offen sind“", bedauert Rajamohan. Ein zweites Problem ist die finanzielle Nachhaltigkeit: In den Zentren sind die Dienstleistungen gratis, ausser Surfen und Spielen auf dem Internet, Telefonanrufe und das Ausdrucken der Dokumente. Diese Einkünfte sind aber sehr mager, und die Stiftung kann nicht damit rechnen, jedes Jahr einen Preis zu erhalten (Sie erhielt 1999 von Motorola einen Preis über 200'000 Dollar). Für K.G. Rahamohan ist deshalb klar: „Die Unterstützung der Stiftung, welche die Löhne der vier Festangestellten des Hubs bezahlt, wird noch lange Zeit notwendig bleiben." 